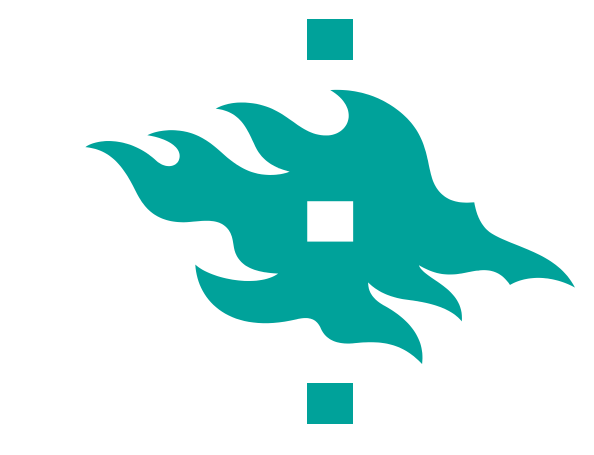

UNIVERSITY OF HELSINKI

FACULTY OF MEDICINE

\title{
Estradiol and follicle stimulating hormone increase cell viability in adult-type ovarian granulosa cell tumor cells - implications for hormonal therapy
}

Ulla-Maija Haltia1 ${ }^{1}$, Marjut Pihlajoki², Noora Andersson'², Lotta Mäkinen ${ }^{1}$, Johanna Tapper $^{1}$, Hugo.M. Horlings ${ }^{3}$, Ursula Turpeinen ${ }^{4}$, Mikko Anttonen ${ }^{4}$, Ralf Bützow ${ }^{5}$, Leila Unkila-Kallio $^{1}$, Olli Carpen ${ }^{5}$, David B Wilson ${ }^{6}$, Markku Heikinheimo ${ }^{2}$ and Anniina Färkkilä1,7

1 University of Helsinki and Helsinki University Hospital, Helsinki, Finland, Obstetrics and Gynecology ${ }^{2}$ University of Helsinki, Children's hospital ${ }^{3}$ The Netherland Cancer Institute, Amsterdam, the Netherlands, ${ }^{4}$ HUSLAB, Helsinki, Finland, 5 University of Helsinki, Pathology, ${ }^{6}$ Washington University School of Medicine, St. Louis, MI, USA, ${ }^{7}$ Radiation Oncology, Harvard Medical School, Boston, MA, USA

\section{Introduction}

Adult-type granulosa cell tumors (AGCTs) are sex-cord derived ovarian malignant tumors. In advanced disease new treatments are needed, as traditional chemotherapeutics have only limited efficacy. Hormonal treatments have been empirically used in treatment of recurrent AGCTs, even though the biological basis for this remains unclear. We aimed to characterize the hormone receptor expression levels in AGCTs and study the effects of estradiol and FSH on AGCT cell growth.

\section{Material and methods}

The expressions for FSH receptor (FSHR), aromatase (CYP19A1) and estrogen receptors (ER $\alpha, E R \beta$ and GPER) were studied in AGCT tissue microarray (175 samples) using immunohistochemistry and RNA in situ method. The effects of FSH and E2 stimulation were assessed in two cell models, established AGCT cell line KGN and primary cultured AGCT cells from six AGCTs. The expression of CYP19A1 in cell lysates were analyzed by RT-PCR. Cell culture medium was collected and analyzed for E2 concentration using mass spectrophotometer. Cell viability was assessed by WST-1.

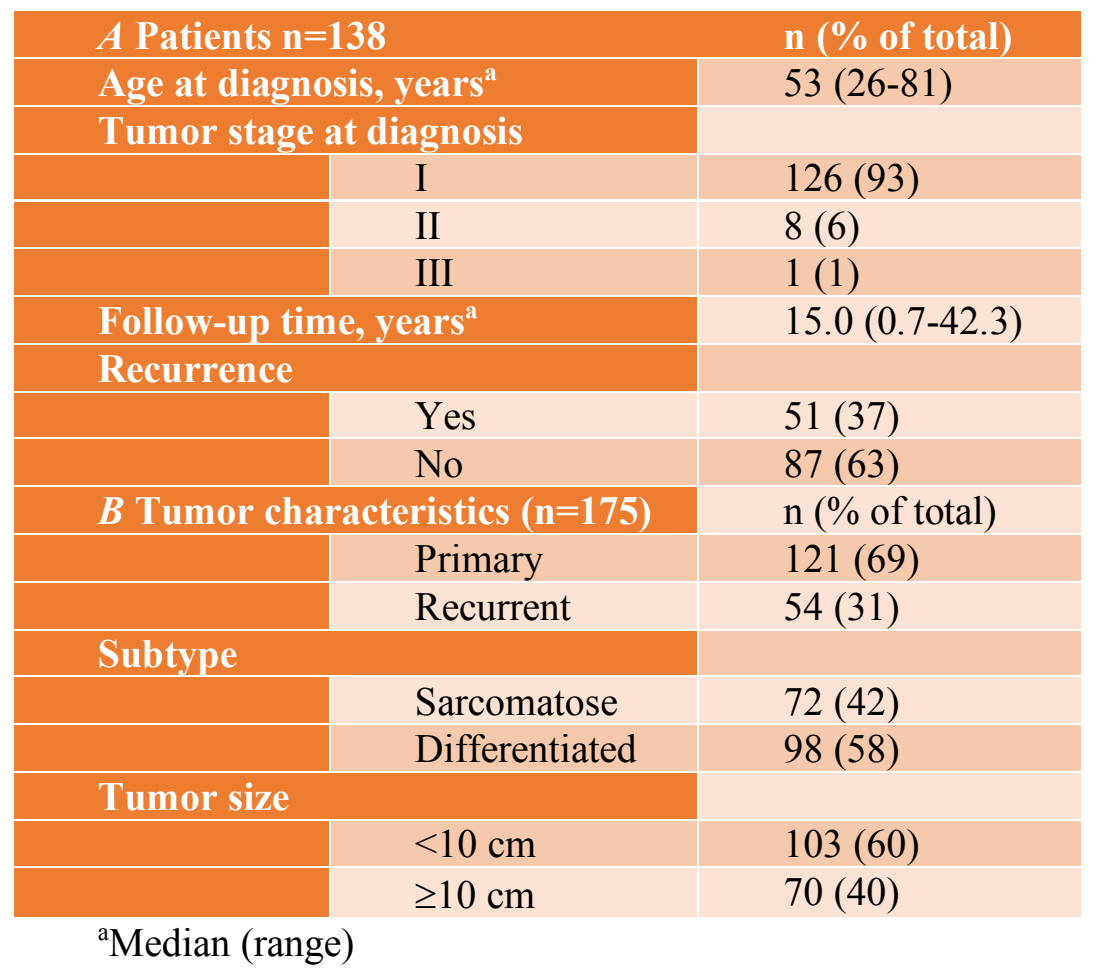

Table 1: Patient data in tumor microarray used in expression analyses. All tumors are tested positive for FOXL2-mutation.

\section{Results}

Figure 1:

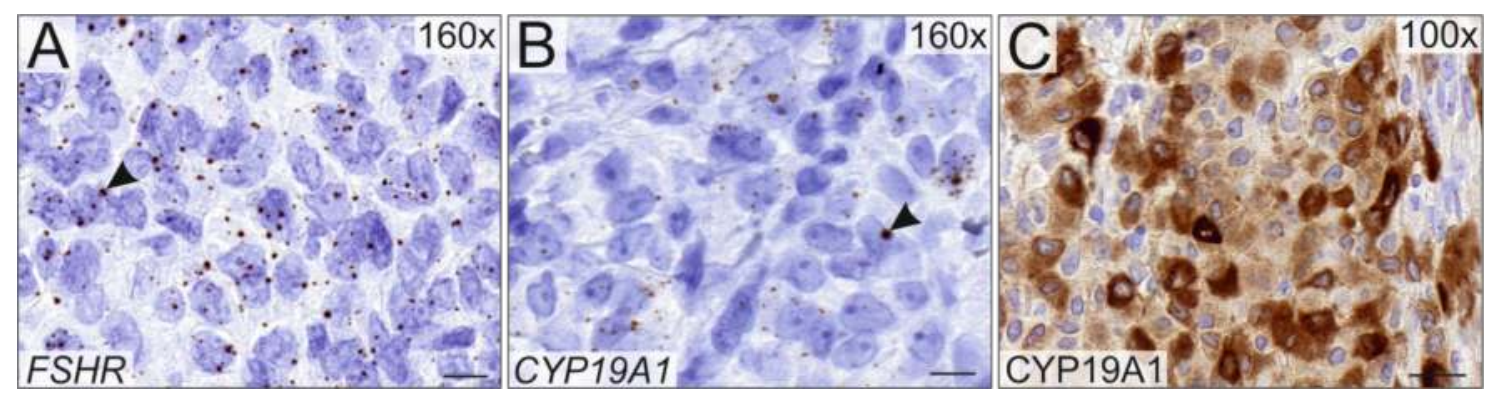

A) FSHR is widely expressed in AGCTs. Expression was detected in $90 \%$ of AGCTs, in $60 \%$ of tumors staining was strong or moderate.

B-C) CYP19A1 is expressed in $48 \%$ of AGCTs. (B: RNA expression, C: protein expression)

Figure 2:
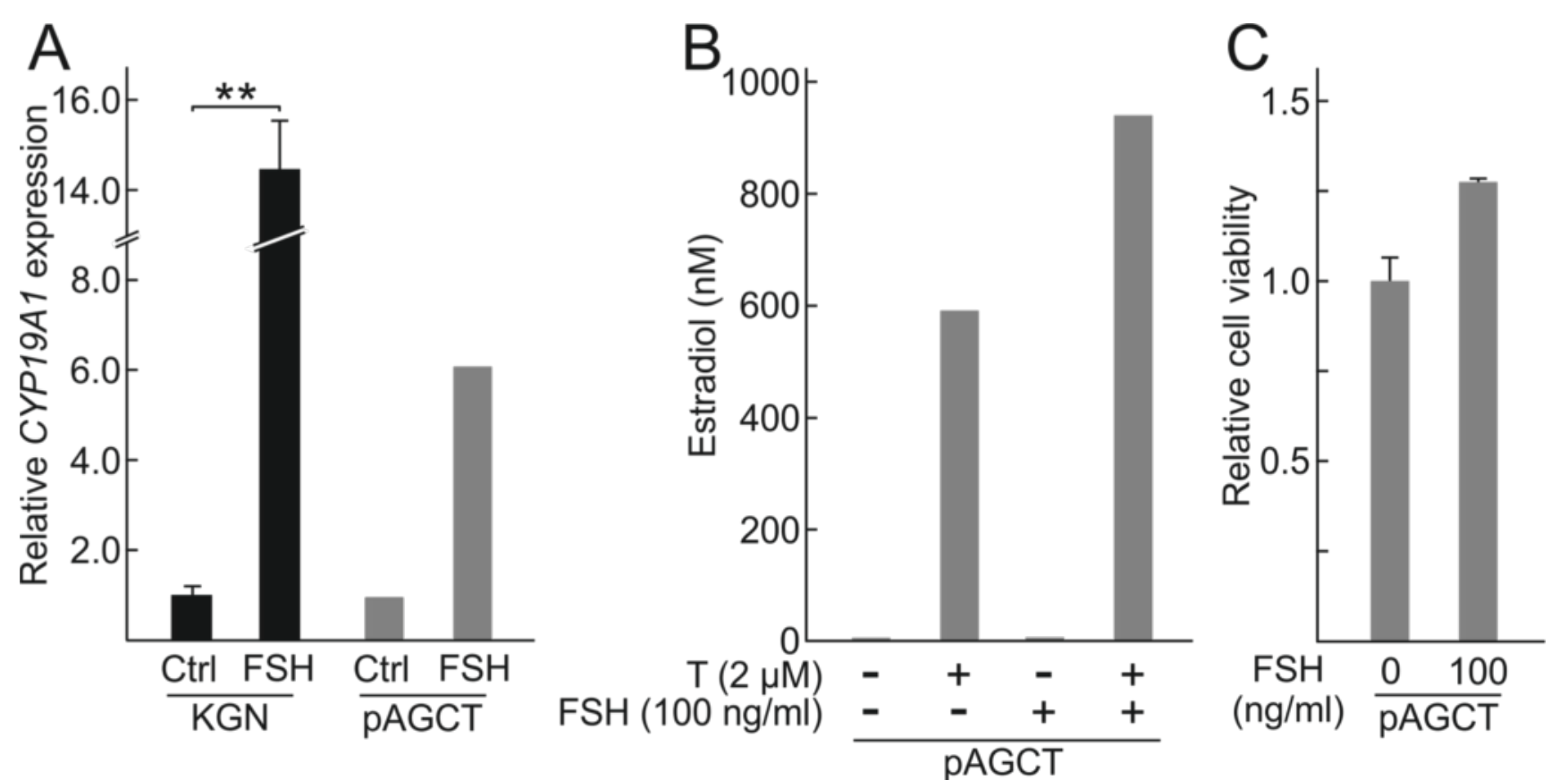

A-C) FSH increases CYP19A1 mRNA expression and E2 production in AGCT cells in vitro and increases cell viability in subset of AGCTs.

Figure 3:

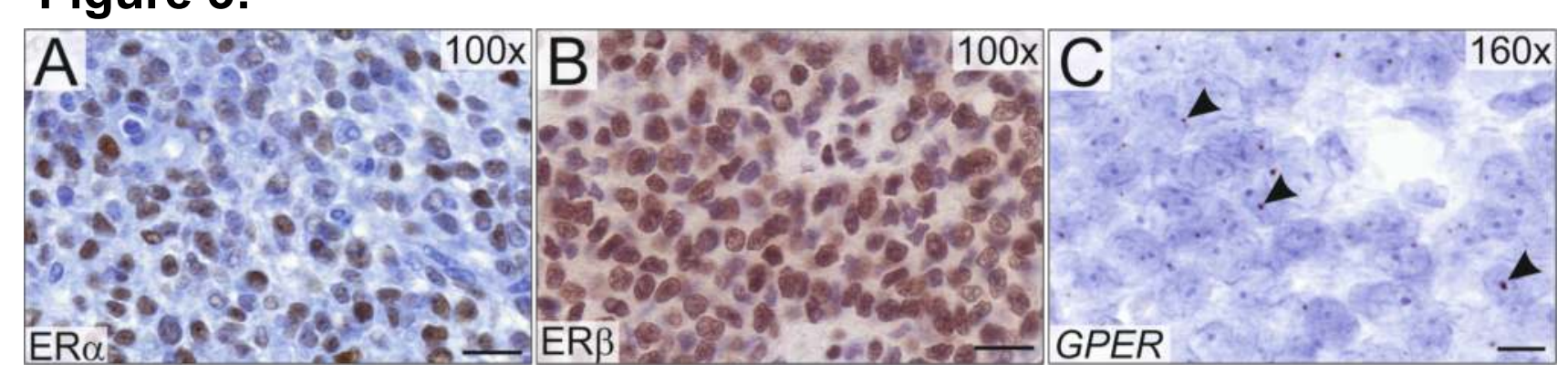

A-C) ER $\beta$ is the main estrogen receptor in AGCTs, while ER $\alpha$ and GPER are expressed in only minority.

ER $\alpha$ in $35 \%$ of AGCTs

ER $\beta$ in $94 \%$ of AGCTs

GPER in $14 \%$ of AGCTs

Figure 4:

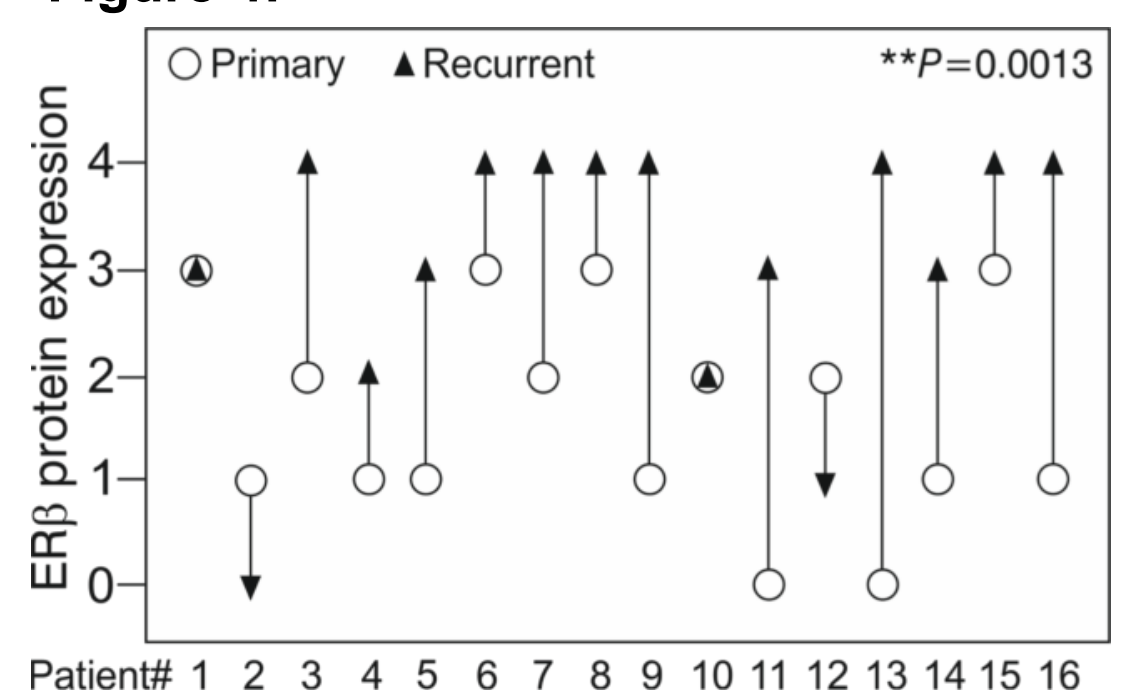

Intensity of ER $\beta$ expression is increased in recurrent tumors.
Figure 5:

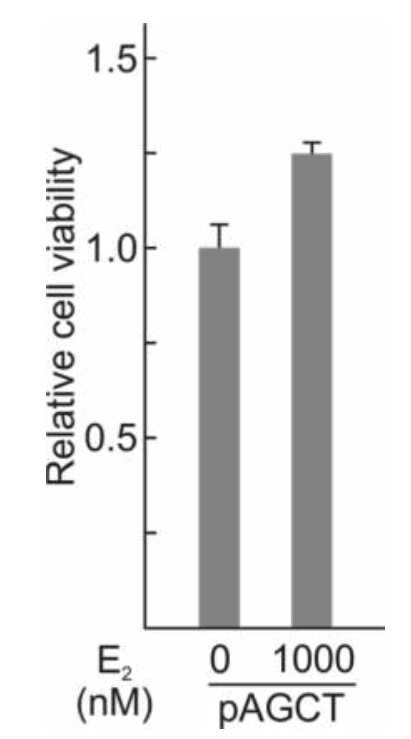

E2 increases cell viability in primary cultured AGCT cells.

\section{Conclusions}

FSH induces CYP19A1 in AGCTs and increases estradiol production and cell viability in AGCTs. ER $\beta$ is the main estrogen receptor in AGCT and its expression is increased in recurrent tumors. Estradiol increases cell viability in AGCTs. 